\title{
Quaternion Affine Moment Invariants for Color Texture Recognition
}

\author{
F. Bouyachcher \\ AGA Lab., University Mohammed First of Oujda, Morocco \\ Université Mohamed 1er, Faculté des Sciences, Département de Maths. et Info., \\ Bd Mohamed VI, B.P. 717, 60000 Oujda, Maroc. \\ B. Bouali \\ AGA Lab., University Mohammed First of Oujda, Morocco \\ Université Mohamed 1er, Faculté des Sciences, Département de Maths. et Info., \\ Bd Mohamed VI, B.P. 717, 60000 Oujda, Maroc. \\ M. Nasri \\ MATSI Lab.,University Mohammed First of Oujda, Morocco
}

\begin{abstract}
We introduce a generalization of the affine moment invariants for color textures recognition using Quaternion Algebra and present results of experiments on a challenging dataset. The advantage of the proposed method is that it can process color image directly, without losing color information. It is shown that the QAMIs can be obtained from the usual AMIs. Experiments show that color texture descriptors are more efficient and discriminant. Moreover, a new set of invariants can be obtained from each QAMI (due to the non-commutativity of Quaternion algebra).
\end{abstract}

\section{General Terms:}

Color image, Invariants

\section{Keywords:}

Quaternion algebra, Affine Moments, Invariants, Color image, Texture

\section{INTRODUCTION}

The theory of moment invariants has been used extensively and many types of moment functions were concerned for image recognition. In the literature, several works dealt with invariant multi-channel objects recognition by transforming the color image into gray-scale one or decomposing the color image into three channels and then computing the moment invariants of the three channels separately [1-4]. But with those methods color information may be lost.

A more interesting approach consists on processing the color image directly in a global manner. Recently, the quaternion algebra has been exploited in color image processing more and more by encoding the three components as a pure quaternion, which allow treating color image as a vector field [5].
The use of the quaternion valued moment functions has been proposed by [6-8]. In this paper we focus on invariants under affine transformations and we introduce a generalization of the affine moment invariants for color images using Quaternion algebra. Quaternion Affine moment invariants (QAMIs) to recognize affine-deformed color images were proposed.

The invariants derived in this study are shown useful and efficient in a numerical example.

\section{PRELIMINARIES}

\subsection{Quaternion algebra}

Quaternions are an extension of the complex numbers, were introduced by Hamilton in 1843 [9]. The set of quaternions form a non commutative ring.

A quaternion is written as the sum of a scalar part and a vector part which consists on three imaginary components as follows

$$
q=a+b i+c j+d k
$$

Where $a, b, c, d \in \mathbb{R}, i^{2}=j^{2}=k^{2}=-1$ and $i j=-j i=$ $k, j k=-k j=i, k i=-i k=j$.

The conjugate of a quaternion $q$ is $\bar{q}=a-b i-c j-d k$.

The modulus of a quaternion $q$ is $|q|=\sqrt{a^{2}+b^{2}+c^{2}+d^{2}}$.

\subsection{Affine moment invariants}

The affine moment invariants are invariant under the following general affine transformation

$$
\begin{aligned}
& u=a_{0}+a_{1} x+a_{3} y, \\
& v=b_{0}+b_{1} x+b_{3} y .
\end{aligned}
$$


The general two dimensional $(p+q)^{t h}$ order moments of a function $f \in L^{2}\left(\mathbb{R}^{2} ; \mathbb{R}\right)$ are defined as:

$$
m_{p q}=\iint_{\propto}^{\infty} x^{p} y^{q} f(x, y) d x d y \quad p, q \in \mathbb{N}
$$

The corresponding central moments are defined as :

$$
\mu_{p q}=\iint_{\propto}^{\infty}\left(x-x_{c}\right)^{p}\left(y-y_{c}\right)^{q} f(x, y) d x d y \quad p, q \in \mathbb{N}
$$

Where $x_{c}=\frac{m_{10}}{m_{00}}$

and $y_{c}=\frac{m_{01}}{m_{00}}$ are the coordinates of the center of gravity of a given object.

In order to achieve the translation invariance T.Suk propose in [10] to use the central moments Equation (3) instead of the general moments Equation (2). The first few affine moment invariants under the general affine transformations Equation (1) of a function $f \in L^{2}\left(\mathbb{R}^{2} ; \mathbb{R}\right)$ introduced in e.g. [10-12] are as follows:

$$
\begin{aligned}
I_{1} & =\left(\mu_{20} \mu_{02}-\mu_{11}^{2}\right) / \mu_{00}^{4}, \\
I_{2} & =\left(-\mu_{30}^{2} \mu_{03}^{2}+6 \mu_{30} \mu_{21} \mu_{12} \mu_{03}-4 \mu_{30} \mu_{12}^{3}-4 \mu_{12}^{3} \mu_{30}\right. \\
& \left.+3 \mu_{21}^{2} \mu_{12}^{2}\right) / \mu_{00}^{10}, \\
I_{6} & =\left(\mu_{40} \mu_{04}-4 \mu_{31} \mu_{13}+3 \mu_{22}^{2}\right) / \mu_{00}^{6} .
\end{aligned}
$$

\section{QUATERNION AFFINE MOMENT INVARIANTS (QAMIS)}

Motivated by processing color image directly without decomposing it on three channels, we define the quaternion-based affine moment invariants. The definition of the Quaternion affine moments is given as follow:

DEFINITION 1. Let $f \in L^{2}\left(\mathbb{R}^{2} ; \mathbb{H}\right)$, the general quaternion moments of $f$ are the quaternion valued functions $m_{Q_{p q}}$ defined by

$$
m_{Q p q}^{(f)}=\iint_{\propto}^{\infty} x^{p} y^{q} f(x, y) d x d y \quad p, q \in \mathbb{N}
$$

DEFINITION 2. Let $f \in L^{2}\left(\mathbb{R}^{2} ; \mathbb{H}\right)$, the central quaternion moments of $f$ are the quaternion valued functions $m_{Q_{p q}}$ defined by

$$
\mu_{Q_{p q}}^{(f)}=\iint_{\propto}^{\propto}\left(x-\left|x_{Q_{c}}\right|\right)^{p}\left(y-\left|y_{Q_{c}}\right|\right)^{q} f(x, y) d x d y \quad p, q \in \mathbb{N}
$$

With $x_{Q_{c}}=\frac{m_{Q_{10}}}{m_{Q_{00}}}=m_{Q_{10}} \cdot \frac{m_{\bar{Q}_{00}}}{\left|m_{Q_{00}}\right|^{2}}$

and $y_{Q_{c}}=\frac{m_{Q_{01}}}{m_{Q_{00}}}=m_{Q_{10}} \cdot \frac{m_{Q_{00}}}{\left|m_{Q_{00}}\right|^{2}}$ are the quaternion coordinates of the center of gravity of $f$.

It follows the definition of the quaternion affine moment invariants.

In order to have invariance of a function $F_{Q}$ of quaternion moments (assuming that $F_{Q}$ has the form of polynomial of quaternion-valued moments) under the general affine transformations Equation (1), as the same way as for usual affine moment invariants [10], invariance of $F_{Q}$ is constructed as follows:

(1) Central quaternion moments are used instead of general quaternion moments for translation invariance. For moments we have :

$$
\mu_{Q_{p q}}^{\prime(f)}=\mu_{Q_{p q}}^{(f)}
$$

With $\mu_{Q_{p q}}^{\prime(f)}$ are the central quaternion moments after translation by given parameters.

(2) $F_{Q}$ is divided by the appropriate power of $\mu_{Q_{00}}^{(f)}$ for two-axis scaling invariance. For moments we have :

$$
\mu_{Q_{p q}}^{(f)}=\omega^{-(p+q+2)} \mu_{Q_{p q}}^{(f)} \text { and } \mu_{Q_{00}}^{\prime(f)}=\omega^{-2} \mu_{Q_{00}}^{(f)},
$$

So,

$$
\frac{\mu_{Q_{p q}}^{\prime(f)}}{\mu_{Q_{00}}^{(f)}{ }^{\frac{p+q}{2}}+1}=\frac{\mu_{Q_{p q}}^{(f)}}{\mu_{Q_{00}}^{(f)}{ }^{\frac{p+q}{2}}+1} .
$$

With $\mu_{Q_{p q}}^{(f)}$ the central quaternion moments after two-axis scaling by the factor $\omega$.

(3) For invariance under one-axis scaling by the factor $\delta$, members of $F_{Q}$ must be isobars. For quaternion valued moments we obtain:

$$
\mu_{Q_{p q}}^{(f)}=\delta^{-(p+1)} \mu_{Q_{p q}}^{(f)} .
$$

We obtain the condition that the sum of $p^{\text {th }}$ indexes of each member of $F_{Q}$ must be equal to the sum of $q^{t h}$ indexes.

(4) Computation of the $F_{Q}$ invariance under skew transformation is obtained as the same way as for usual affine moment invariants [10], with replacing the real valued function by quaternion valued function.

One can see that conditions to have invariance of quaternion affine moments are the same conditions to have invariance of usual affine moments, hence, we can embed usual affine moment invariants introduced by T.Suk in e.g. [10-12] into the quaternion algebra. Since the value of these affine invariants is a quaternion. Two different quaternions cannot be compared directly but this is possible using their magnitude.

We can construct quaternion affine invariants as follows:

$$
I_{Q}=\left|F_{Q}\right| \text {. }
$$

\section{APPLICATION TO COLOR IMAGE RECOGNITION}

\subsection{Quaternion affine moment invariants for color image}

For the application we have in mind the color image recognition, we define quaternion affine moment invariants of a color image. We consider the subset $H_{0}$ of pure quaternions for encoding three-channel images.

DEFINITION 3. Let $f \in L^{2}\left(\mathbb{R}^{2} ; \mathbb{H}\right)$ be a function associated to a color image and defined by

$$
f(x, y)=f_{r}(x, y) i+f_{g}(x, y) j+f_{b}(x, y) k .
$$

Where $f_{r}, f_{r}$ and $f_{r}$ correspond to the red, green and blue levels. The quaternion central moments of $f$ are the quaternion valued functions $\mu_{p q}$ defined by

$$
\mu_{Q_{p q}}^{(f)}=\iint_{\propto}^{\infty}\left(x-\left|x_{Q_{c}}\right|\right)^{p}\left(y-\left|y_{Q_{c}}\right|\right)^{q} f(x, y) d x d y \quad p, q \in \mathbb{N} .
$$

We derive the quaternion affine moment invariants for color images from quaternion affine moment invariants. 


\subsection{Color image recognition I: simple texture images}

We now present application to color image recognition to validate the effectiveness of the proposed QAMIs under affine transformations. The original color images used in tests are shown on Figure 1 (images are downloaded from Mayang Textures Library [13]), all these images are $300 \times 300$ pixels. Figure 2 presents a sample image, showing a textured surface viewed under different transformed version. For experiments the real-valued invariants $I_{Q_{1}}$ and $I_{Q_{6}}$ are used (the user has freedom to choose invariants to use (an optimum choice: depending on number and quality of descriptors)).

$$
\begin{aligned}
& I_{Q_{1}}=\left|\left(\mu_{Q_{20}} \mu_{Q_{02}}-\mu_{Q_{11}}^{2}\right) / \mu_{Q_{00}}^{4}\right| \\
& I_{Q_{6}}=\left|\left(\mu_{Q_{40}} \mu_{Q_{04}}-4 \mu_{Q_{31}} \mu_{Q_{13}}+3 \mu_{Q_{22}}^{2}\right) / \mu_{Q_{00}}^{6}\right| .
\end{aligned}
$$

For the texture recognition experiments, as one can see from Figure 3 . the classification of images was correct with a null error rate; the QAMIs remain almost unchanged for all affine transformations of each image. Therefore, the QAMIs derived here could be useful in color pattern recognition tasks that require affine transformations. Remark Due to the non commutativity of quaternions, every permutation of moments, in the product terms of the invariants, can be considered as a new invariant (we had obtained invariance from experimental tests. We may do the theoretical demonstration in a future work). Here are examples of the two QAMIs used in experiments :

$$
\begin{aligned}
& I_{Q_{1,1}}=\left|\left(\mu_{Q_{20}} \mu_{Q_{02}}-\mu_{Q_{11}}^{2}\right) / \mu_{Q_{00}}^{4}\right| \\
& I_{Q_{1,2}}=\left|\left(\mu_{Q_{02}} \mu_{Q_{20}}-\mu_{Q_{11}}^{2}\right) / \mu_{Q_{00}}^{4}\right| .
\end{aligned}
$$

and

$$
\begin{aligned}
I_{Q_{6,1}} & =\left|\left(\mu_{Q_{40}} \mu_{Q_{04}}-4 \mu_{Q_{31}} \mu_{Q_{13}}+3 \mu_{Q_{22}}^{2}\right) / \mu_{Q_{00}}^{6}\right|, \\
I_{Q_{6,2}} & =\left|\left(\mu_{Q_{04}} \mu_{Q_{40}}-4 \mu_{Q_{31}} \mu_{Q_{13}}+3 \mu_{Q_{22}}^{2}\right) / \mu_{Q_{00}}^{6}\right|, \\
I_{Q_{6,3}} & =\left|\left(\mu_{Q_{40}} \mu_{Q_{04}}-4 \mu_{Q_{13}} \mu_{Q_{31}}+3 \mu_{Q_{22}}^{2}\right) / \mu_{Q_{00}}^{6}\right|, \\
I_{Q_{6,4}} & =\left|\left(\mu_{Q_{04}} \mu_{Q_{40}}-4 \mu_{Q_{13}} \mu_{Q_{31}}+3 \mu_{Q_{22}}^{2}\right) / \mu_{Q_{00}}^{6}\right| .
\end{aligned}
$$

\subsection{Color image recognition II: challenging dataset}

In this section, we show classification results on a challenging dataset (Figure 4), it contains 4 different texture classes (ground (A, B and C) and wool (L)) where each class is represented by one image divided into 40 sub-images of $50 \times 50$ pixels. And here also, the real-valued invariants $I_{Q_{1}}$ and $I_{Q_{6}}$ are used for experimental tests. Figure 5 shows the category classification results. We can observe that the proposed method works well, textures are almost correctly classified. Table 1 shows the confusion matrix and the classification rates for the individual classes. Experiments on the 4 texture classes demonstrate the effectiveness of the QAMIs for visual classification.

\section{CONCLUSION}

In this paper we have extended the usual affine moments to color image using the quaternion algebra. The quaternion based moment invariants with respect to affine transformations have been constructed. The experiment results show that the proposed method is efficient and perform better the existing one which is based on RGB decomposition. The main advantage of the QAMIs is that it can treat color image in a global manner and directly, without losing color information.
Future works will be devoted to find a general form of the affine moment invariants for multi-channel objects (with more than three levels) using Clifford algebra.

\section{References}

[1] Y.K. Chan and C.C. Chang. A color image retrieval method based on color moment and color variance of adjacent pixels. Int. J. Pattern Recognition. Artif. Intell., 16:113-125, 2002.

[2] F. Mindru, T. Moons, and L.V. Gool. Color-based moment invariants for the viewpoint and illumination independent recognition of planar color patterns. In Int. Conf. Advances in Pattern Recognition (ICAPR'98), pages 113-122, 1998.

[3] F. Mindru, T. Tuytelaars, L.V. Gool, and T. Moons. Moment invariants for recognition under changing viewpoint and illumination. Comput. Vis. Image Und., 94:3-27, 2004.

[4] T. Suk and J. Flusser. Affine moment invariants of color images. In CAIP 2009, LNCS5702, pages 334-341, 2009.

[5] T.A. Ell and S.J. Sangwine. Hypercomplex fourier transforms of color images. IEEE Trans. Image Process., 16:22-35, 2007.

[6] B.J. Chen, H.Z. Shu, H. Zhang, G. Chen, J.L. Dillenseger, and L.M. Luo. Quaternion zernike moments and their invariants for color image analysis and object recognition. Signal Processing, 92:308-318, 2012.

[7] B.J. Chen, H.Z. Shu, H. Zhang, G. Chen, and L.M. Luo. Color image analysis by quaternion zernike moments. In 20th International Conference on Pattern Recognition (ICPR2010), pages 625-628, 2010.

[8] L.Q. Guo and M. Zhu. Quaternion fourier-mellin moments for color image. Pattern Recognition, 44:187-195, 2011.

[9] W. R. Hamilton. Elements of quaternions. Longmans Green, London, U.K., 1866.

[10] J. Flusser and T. Suk. Pattern recognition by affine moment invariants. Pattern Recognition, 26:167-174, 1993.

[11] J. Flusser and T. Suk. Tables of affine moment invariants generated by the graph method. Technical report, Institute of information Theory and Automation, 2005.

[12] T. Suk and J. Flusser. Graph method for generating affine moment invariants. In 17th Int. Conf. on Pattern Recognition, volume 2, pages 192-195,2004.

[13] Mayang textures library. http://www.mayang.com/textures/. 


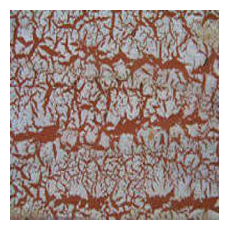

Cracked paint

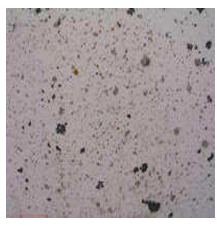

Painted surface dirt flecks

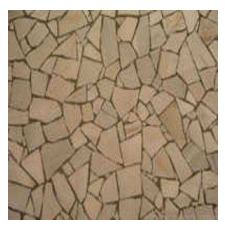

Crazy paving

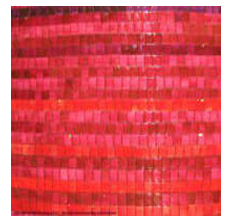

Red shiny wet tiles

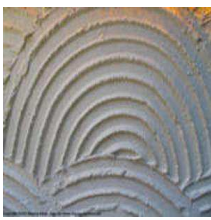

Grout paint

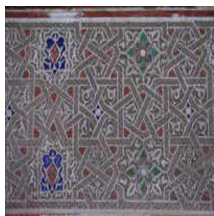

Traditional islamic plasterwork

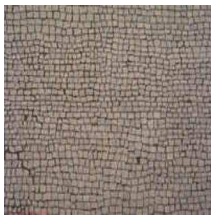

Mosaic tiles

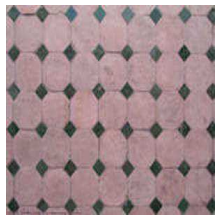

Weathered tiles

Fig. 1. The original images used in experiment tests
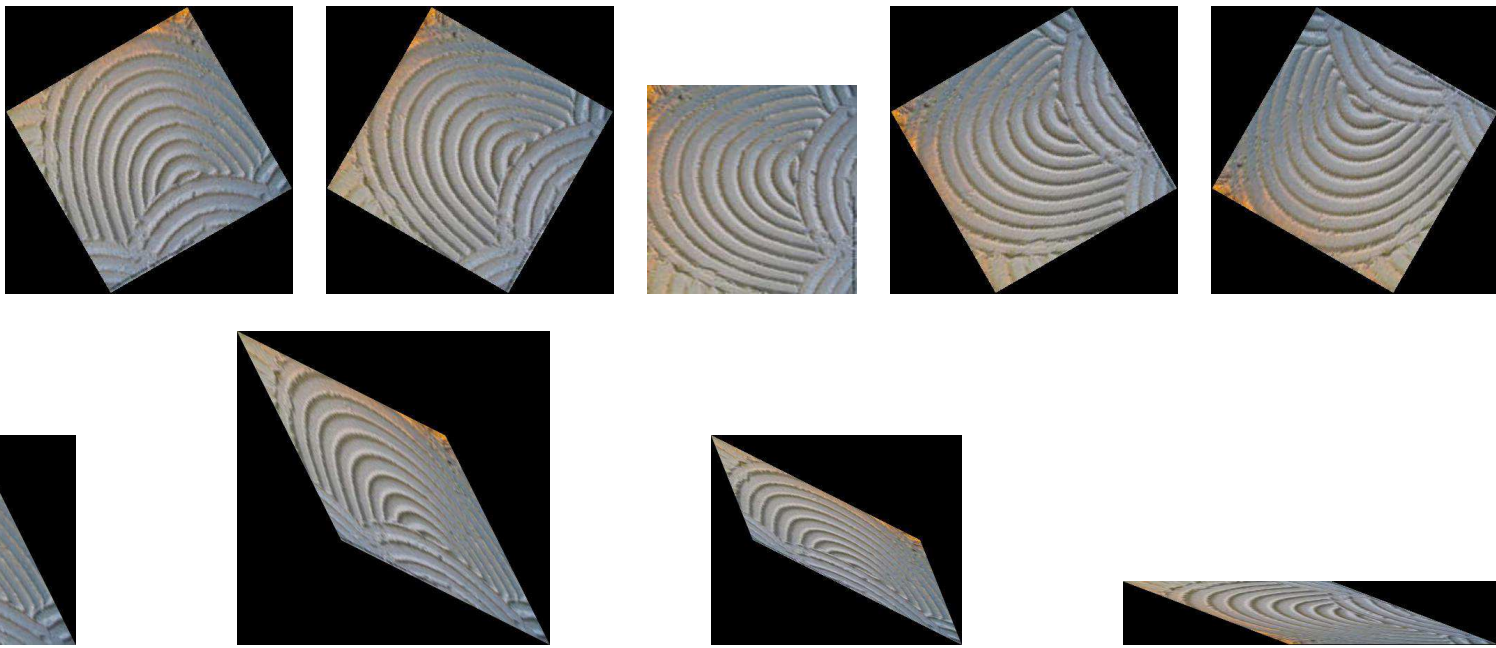

Fig. 2. The original color image -grout paint- and its transformed version

Table 1. Confusion matrix for the dataset used in Subsection 4.3

\begin{tabular}{|c|c|c|c|c|c|}
\hline Classes & A & B & C & L & Rate \\
\hline A & 31 & 0 & 9 & 0 & 77.5 \\
\hline B & 0 & 22 & 17 & 1 & 55 \\
\hline C & 3 & 7 & 30 & 0 & 75 \\
\hline L & 0 & 0 & 0 & 40 & 100 \\
\hline
\end{tabular}




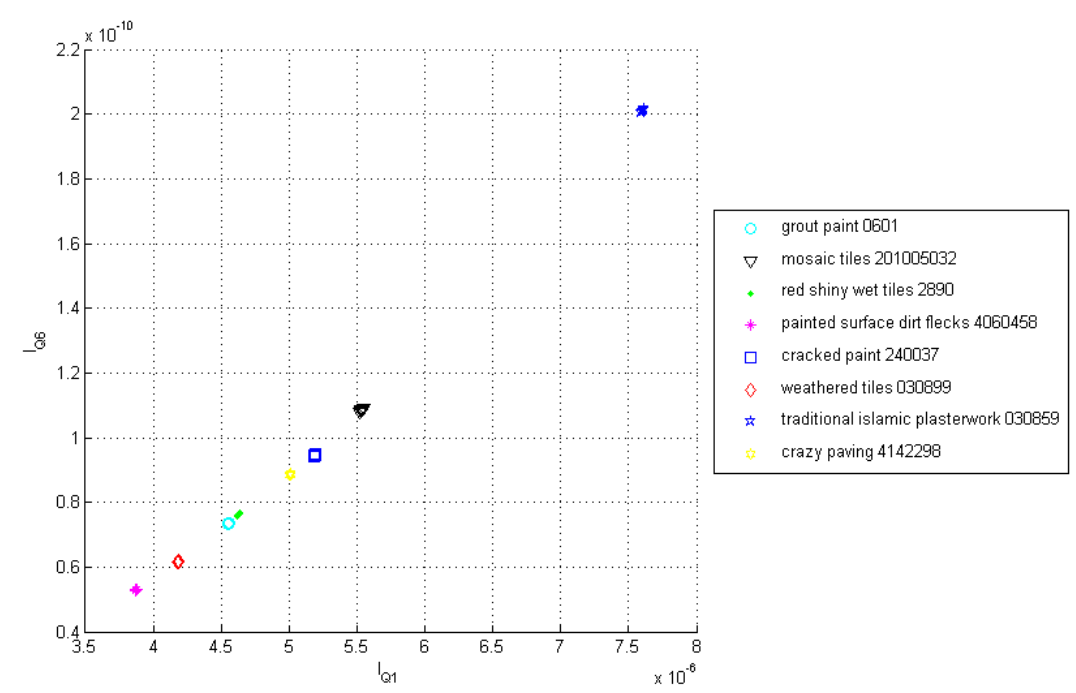

Fig. 3. Texture classification results (I)

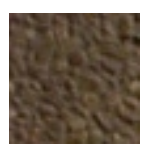

A1

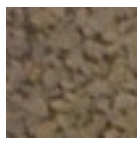

B1

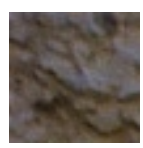

C1

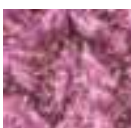

L1

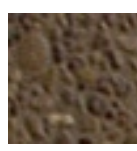

A2

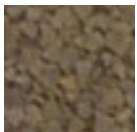

B2

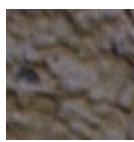

$\mathrm{C} 2$

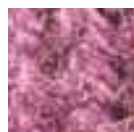

L2

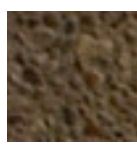

A3

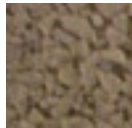

B3

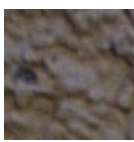

C3

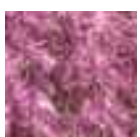

L3

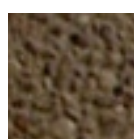

A4

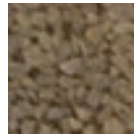

B4

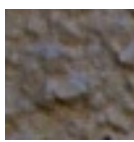

$\mathrm{C} 4$

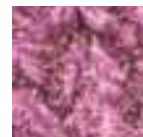

L4

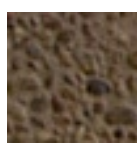

A5

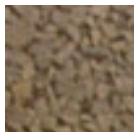

B5

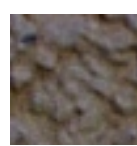

C5

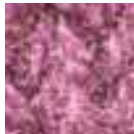

L5

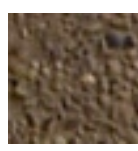

A6

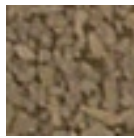

B6

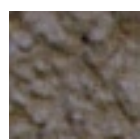

C6

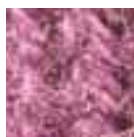

L6

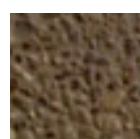

A7

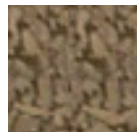

B7

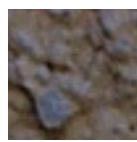

C7

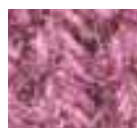

L7

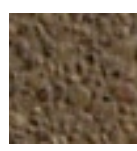

A8

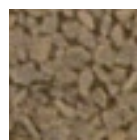

B8

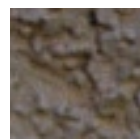

$\mathrm{C} 8$

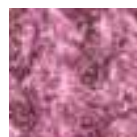

L8

Fig. 4. Samples of the dataset used in Subsection 4.3 experiment tests 


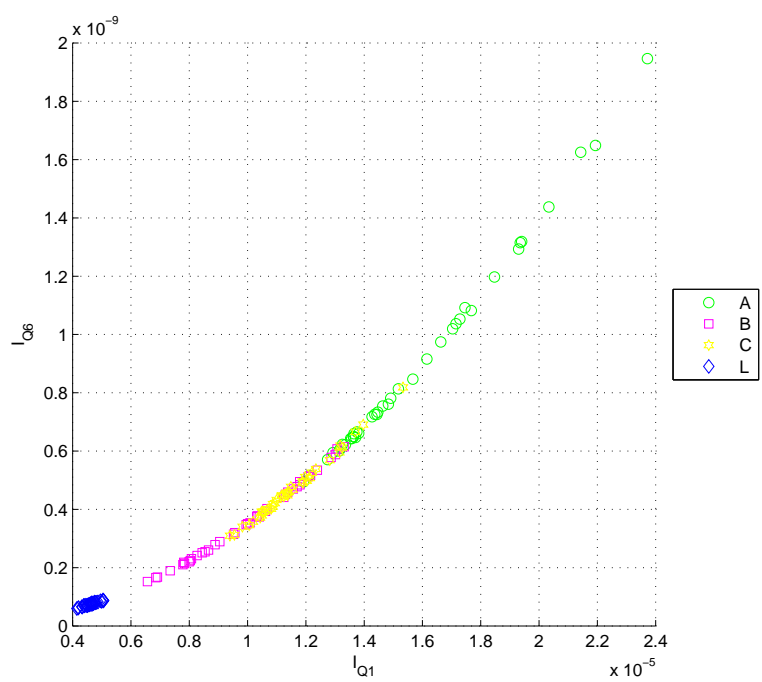

Fig. 5. Texture classification results (II) 